Supporting Information for

\title{
Optical Multilevel Spin Bit Device using Chiral Quantum Dots
}

\author{
H. Al-Bustami, ${ }^{1}$ B. P. Bloom, ${ }^{2}$ Amir Ziv, ${ }^{1}$ S. Goldring, ${ }^{1}$ S. Yochelis, ${ }^{1}$ R. Naaman*, ${ }^{3}$ D. H, Waldeck*, ${ }^{2}$ Y. \\ Paltiel*1
}

\footnotetext{
1. Applied Physics Department and the Center for Nano-Science and Nano-Technology, The Hebrew University of Jerusalem, Jerusalem, 91904 Israel.

2. Chemistry Department, University of Pittsburgh, Pittsburgh PA 15260 USA

3. Department of Chemical and Biological Physics, Weizmann Institute, Rehovot, 76100 Israel
}

\section{Content}

Fabrication of Hall Device, Synthesis of QDs, Hall device assembly, Characterization of LCP and RCP, Stability of the $\mathrm{Al}_{2} \mathrm{O}_{3}$ capped devices, Full nine level logic operation, Effect of linear polarization

\section{Fabrication of Hall Device}

Every chip contains two Hall sensors. Each chip is processed through two fabrication steps before the adsorption.

The large contacts (Figure S1, yellow bold lines and pads) are fabricated using an optical lithography process, that contains:

- Chip cleaning (sonication for $5 \mathrm{~min}$ in each of acetone, methanol, and isopropanol).

- Spin coating of lift-off resist (LOR) followed by AZ1512 positive resist@4000 rpm.

- Exposure process using laser writer.

- Gold deposition using Evaporation.

- Metal liftoff in hot N-methyl2-pyrrolidone (NMP) bath.

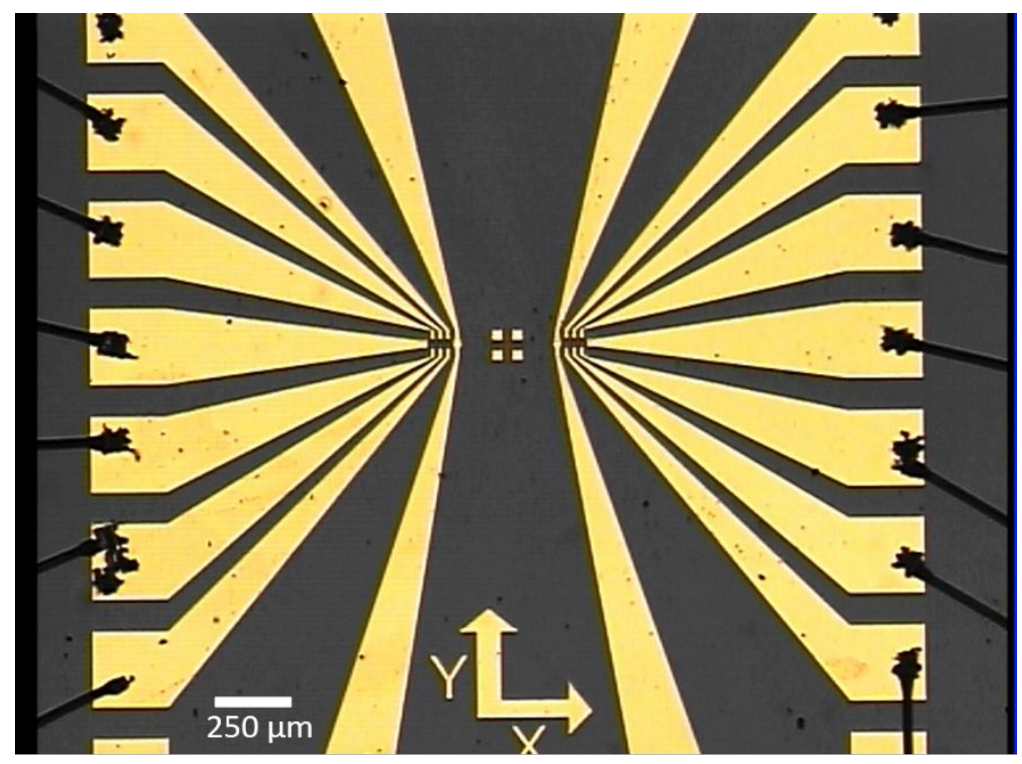

Figure S1: Optical microscope image of the Hall sensor 
The Hall bar was fabricated using optical lithography by the following steps:

- Sample cleaning hot acetone for $5 \mathrm{~min}$ following by hot ethanol for $5 \mathrm{~min}$.

- Spin coating of LOR followed by positive resist (the same parameters as first process).

- Exposure process using laser writer.

- Metal deposition of $5 \mathrm{~nm}$ ferromagnetic Ni followed by $5 \mathrm{~nm}$ Au as passivation layer.

- Lift-off of the metals.

\section{Synthesis of QDs}

Achiral octadecylphosphonic acid capped QDs were first synthesized following previously published protocols. ${ }^{1}$ The purified CdSe QDs were then ligand exchanged with either chiral (Lcysteine or D-cysteine) or achiral (mercaptoproprionic acid) ligands using a modified procedure developed by Tohgha et al.. ${ }^{2}$ Briefly, a $2.0 \mathrm{~mL}$ solution of water with $82.5 \mathrm{mmol}$ of the new ligand was adjusted to a $\mathrm{pH}$ of 11 using concentrated tetramethylammonium hydroxide. Next, $2.0 \mathrm{~mL}$ of an $82.5 \mu \mathrm{M}$ CdSe QD solution in chloroform was added to the water solution and mixed under argon in the dark overnight. The QDs transferred to the aqueous phase upon successful ligand exchange. The resulting solution was then centrifuged and the aqueous layer was collected. The QDs were purified using a syringe and centrifugal filter. Precipitation and centrifugation of the QDs was carried out using isopropyl alcohol and the resulting pellet was dried with a stream of argon for storage.

\section{Hall device assembly}

The adsorbing procedure of the Chiral QDs was done in several stages. First, the device was cleaned with hot acetone then it was processed in an ozonator to remove/oxidize any organic residues and lastly was immersed in absolute ethanol for 20 minutes. The adsorption procedure followed, started with immersing the device in $1 \mathrm{mM}$ Cysteamine ethanol solution overnight. Then the device was transferred to an ethanol solution of the Chiral QDs overnight. Lastly, the device was washed with ethanol and dried under nitrogen. The final device was bonded and kept in nitrogen chamber before measurement.

\section{Characterization of LCP and RCP}

Figure $\mathrm{S} 2$ shows the optical setup with a detection of LCP/RCP using a polarimeter. The right and left circular polarizations were achieved using a half- wave plate and a quarter-wave plate. Here, we use a polarimeter which is constructed along the optical path to detect the efficiency of the polarization. 


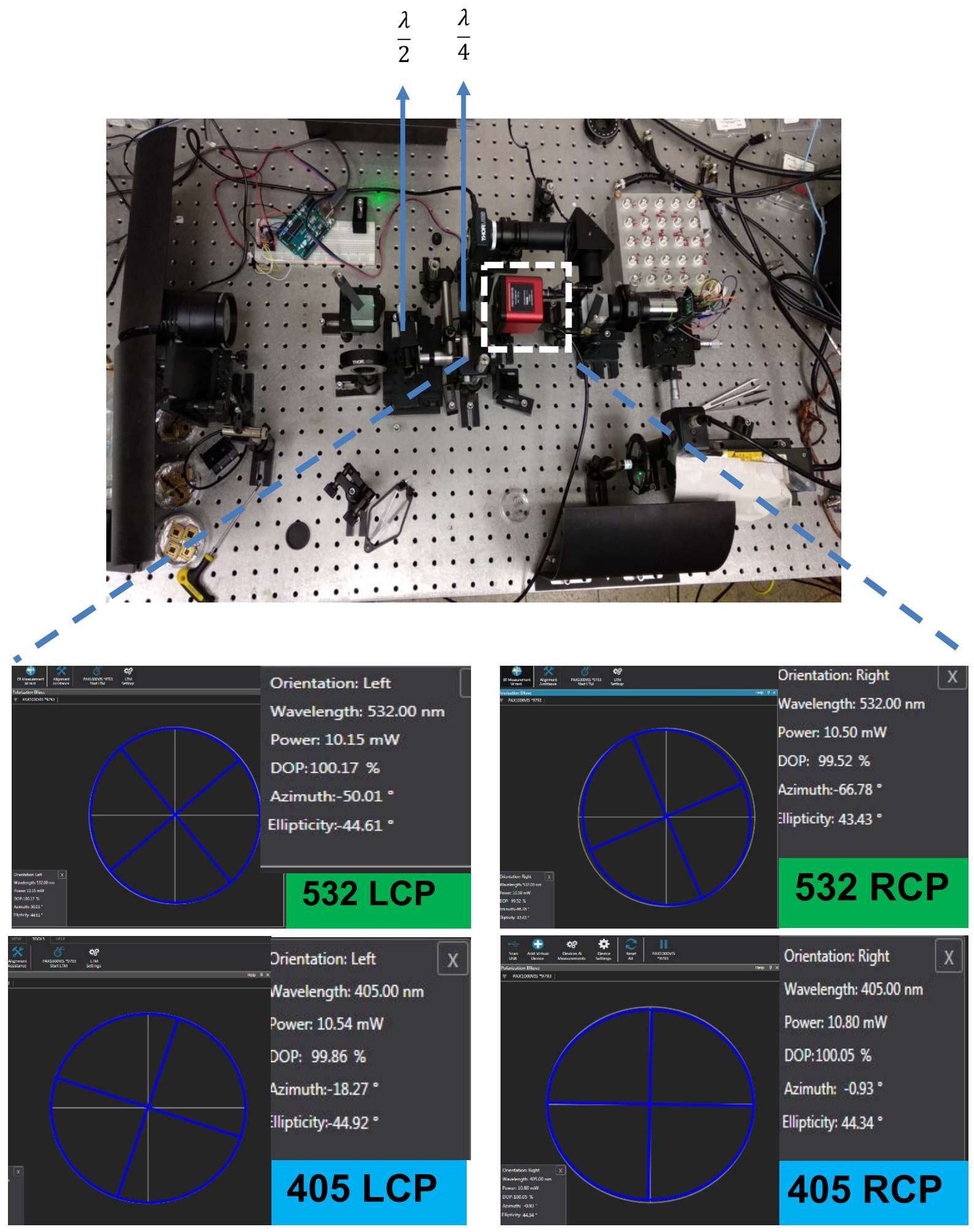

Figure S2: Polarimeter measurements of $532 \mathrm{~nm}$ and $405 \mathrm{~nm}$ lasers.

Stability of the $\mathrm{Al}_{2} \mathrm{O}_{3}$ capped devices 
To prevent oxidation, and subsequent device degradation, the devices were encapsulated with $\mathrm{Al}_{2} \mathrm{O}_{3}$ using atomic layer deposition. Devices that were encapsulated were stored for a month in ambient temperature and humidity in both light and dark conditions. Three samples, each containing two Hall devices, for each chirality possessed long term stability.

Figure S3 shows the Hall measurement of the same devices measured in Figure 4 after 30 days stored under ambient conditions. A similar signal intensity was observed as that which is shown in Figure 4 of the main text.

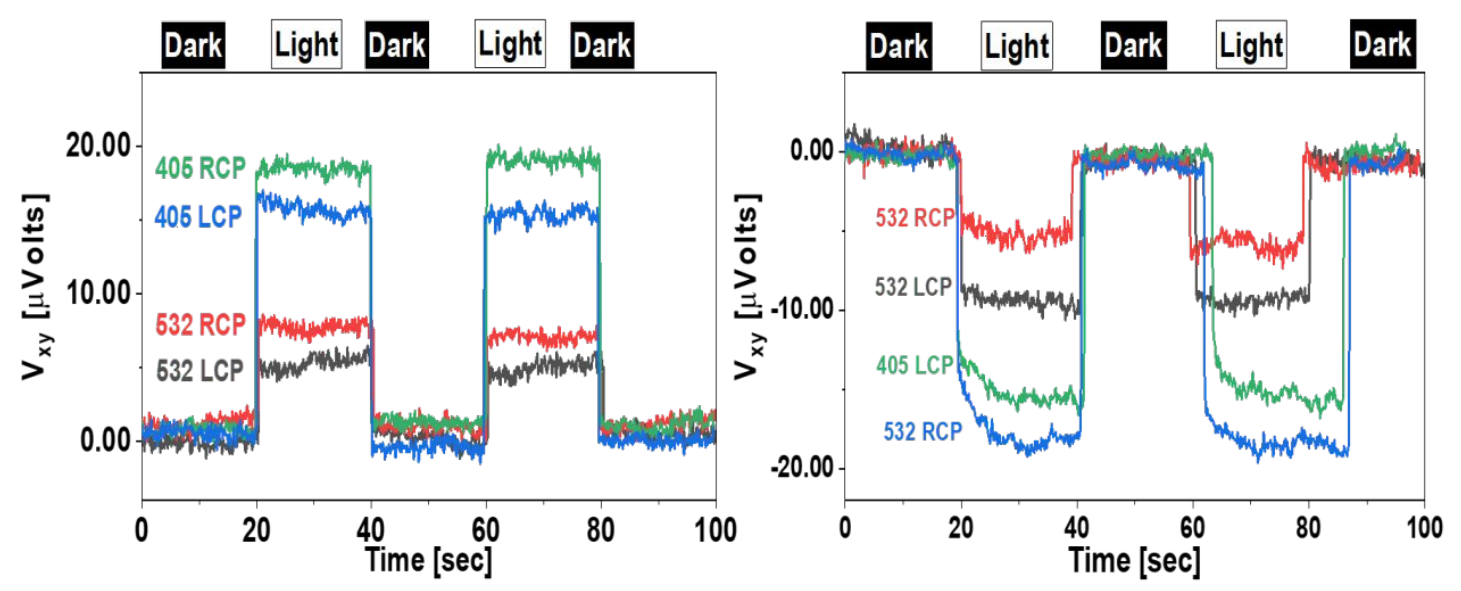

Figure S3: Stability testing of the Device. Hall voltage measurements on the same Hall devices shown in Figure 4 of the main text after storage in ambient conditions for 30 days. The panel on the left is for devices with D-cysteine coated QDs and the panel on the right is for L-cysteine coated QDs (right).

\section{Linear polarization}

The effect of excitation with linear polarized excitation is shown in Figure S5. An intermediate signal intensity to that of RCP and LCP is observed, consistent with what is expected for the CISS effect.

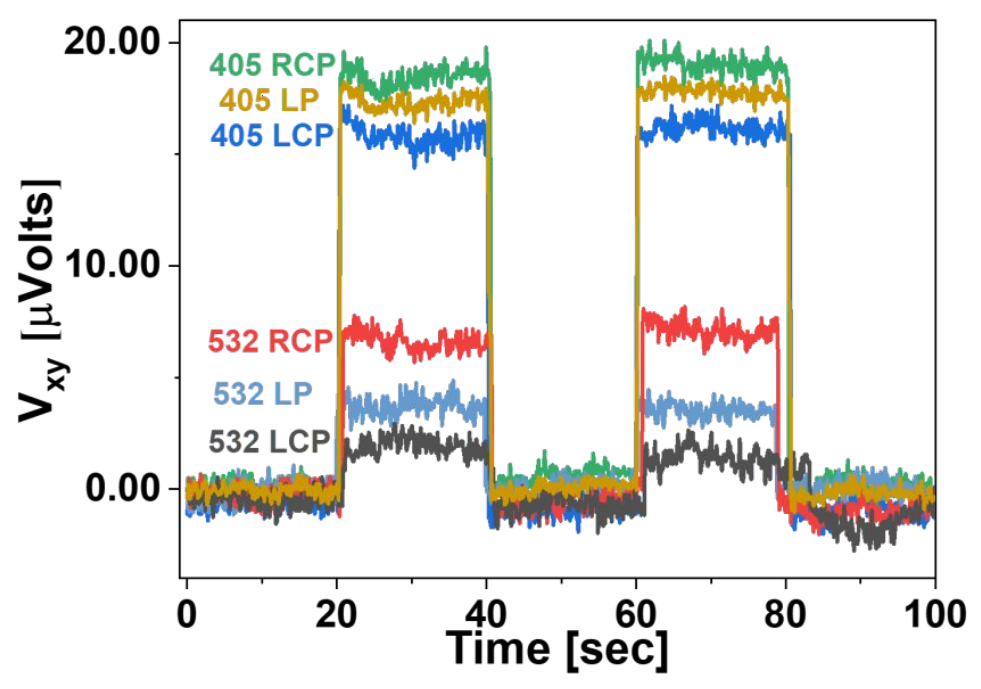

Figure S4: Hall device measurements on D-cysteine coated QDs excited using LCP (Left Circular Polarized Light), RCP (Right Circular Polarized Light) and LP (linear Polarized Light) with $532 \mathrm{~nm}$ and $405 \mathrm{~nm}$ light 
1. Kalyuzhny, G.; Murray, R. W. Ligand effects on optical properties of nanocrystals $J$. Phys. Chem. B 2005, 109, (15), 7012-7021.

2. Tohgha, U.; Deol, K. K.; Porter, A. G.; Bartko, S. G.; Choi, J. K.; Leonard, B. M.; Varga, K.; Kubelka, J.; Muller, G.; Balaz, M. Ligand Induced Circular Dichroism and Circularly Polarized Luminescence in CdSe Quantum Dots ACS Nano 2013, 7, (12), 11094-11102. 